\title{
A Player-Centric Approach to Designing Spatial Skill Training Games
}

\author{
Helen Wauck \\ University of Illinois \\ Urbana-Champaign \\ Urbana, Illinois \\ wauck2@illinois.edu
}

\author{
Elisa D. Mekler \\ University of Basel \\ Basel, Switzerland \\ elisa.mekler@unibas.ch
}

\author{
Wai-Tat Fu \\ University of Illinois \\ Urbana-Champaign \\ Urbana, Illinois \\ wfu@illinois.edu
}

\begin{abstract}
Certain video games show promise as tools for training spatial skills, one of the strongest predictors of future success in STEM. However, little is known about the gaming preferences of those who would benefit the most from such interventions: low spatial skill students. To provide guidance on how to design training games for this population, we conducted a survey of 350 participants from three populations: online college-age, students from a low SES high school, and students from a high SES high school. Participants took a timed test of spatial skills and then answered questions about their demographics, gameplay habits, preferences, and motivations. The only predictors of spatial skill were gender and population: female participants from online and low SES high school populations had the lowest spatial skill. In light of these findings, we provide design recommendations for game-based spatial skill interventions targeting low spatial skill students.
\end{abstract}

\section{CCS CONCEPTS}

-Human-centered computing $\rightarrow$ User studies; User models;

\section{KEYWORDS}

spatial reasoning, cognitive training, gender, video games, emotions, motivation

ACM Reference Format:

Helen Wauck, Elisa D. Mekler, and Wai-Tat Fu. 2019. A PlayerCentric Approach to Designing Spatial Skill Training Games. In CHI Conference on Human Factors in Computing Systems Proceedings

Permission to make digital or hard copies of all or part of this work for personal or classroom use is granted without fee provided that copies are not made or distributed for profit or commercial advantage and that copies bear this notice and the full citation on the first page. Copyrights for components of this work owned by others than ACM must be honored. Abstracting with credit is permitted. To copy otherwise, or republish, to post on servers or to redistribute to lists, requires prior specific permission and/or a fee. Request permissions from permissions@acm.org.

CHI 2019, May 4-9, 2019, Glasgow, Scotland UK

(C) 2019 Association for Computing Machinery.

ACM ISBN 978-1-4503-5970-2/19/05 ..\$15.00

https://doi.org/10.1145/3290605.3300296
(CHI 2019), May 4-9, 2019, Glasgow, Scotland UK. ACM, New York, NY, USA, 13 pages. https://doi.org/10.1145/3290605.3300296

\section{INTRODUCTION}

In the last couple decades, there has been great interest in harnessing the motivational power of video games for learning. A variety of video games have shown promise as educational and cognitive training tools, from games designed to teach math $[41,44,51,59]$, programming $[4,16]$, and history [76], to commercial games repurposed for academic learning $[46,64]$ or cognitive skill training $[6,24,28]$. One particularly strong focus in the research literature is the potential video games have to train a subset of cognitive skills shown to be one of the strongest predictors of success in STEM majors and careers: spatial skills [75]. Certain video games, such as Medal of Honor, Super Mario 64, Crazy Taxi, Zaxxon, and Portal 2, have been shown to train spatial skills in controlled laboratory settings $[18,22,23,33,62,71]$.

However, it is not clear whether these games would be equally effective outside of a laboratory experiment. In a more naturalistic gameplay setting, where players play just for fun and not for monetary compensation or course credit, it is not enough for a game to be empirically effective at training. It must also be enjoyable and entertaining enough that its intended audience will actually play it, and play it long enough to see training effects. But what should the intended audience for a spatial skill training game be?

We argue that students with the lowest levels of spatial skill should be the target audience for spatial skill training games given that they stand the most to benefit from them. Students with low spatial skill tend to struggle in introductory courses and are more likely to drop out of STEM majors - unless they can bring their spatial skills up to a certain "threshold" of ability that gets them through early STEM coursework [70, 71]. This is especially a problem for female students as research has shown that females consistently perform worse males on spatial skill assessments, beginning as early as elementary school $[35,40,42]$. This may be one reason that the gender gap in many STEM fields remains a problem [39]. Bringing low spatial skill students' spatial skills up to a certain threshold could be one way of reducing 
this gender gap in STEM, and in general allowing more students who otherwise might drop out to continue in STEM majors and then on to STEM careers in the future.

Unfortunately, most of the games that have been successful at training players' spatial skills in the laboratory may be most appealing to the subset of the population that already has higher levels of spatial skills: male action video game players. Most video games shown empirically to train spatial skills are action games, and those who play action video games more often generally have higher spatial skills $[6,56,60]$. In addition, men and boys tend to enjoy action video games more than women and girls [29, 56, 67, 83], and each of the previously studied games that train spatial skills are commercial games, which for decades have been designed with men and boys as the target audience [14, 31, 55].

In essence, those with low spatial skill, especially women and girls, are not being served by current approaches to finding game-based spatial skill training interventions. Designing training games with low spatial skill students in mind is therefore essential for addressing this problem and helping more underrepresented students pursue STEM careers. The current work takes a player-centered approach $[66,72]$ to designing games for this target population by asking directly for their input about what they like in a gaming experience. Combining this information with demographic characteristics allows us to present a player persona [13] of sorts to help game designers understand the preferences of low spatial skill populations.

We conducted an online and classroom survey across a diverse set of high school students and college age adults $(n=350)$. The survey asked participants to take a test of spatial skills and then fill out a series of questionnaires about their gameplay habits, preferences, and motivations, as well as basic demographic information. In order to encourage participants to take the spatial skills test and survey questions seriously, we gave participants feedback at the end about their performance on the test and their gaming motivation profile based on their survey responses.

Contrary to existing literature, we found no evidence for a relationship between spatial skill and gameplay habits, genre preferences, or motivations. The only strong predictors of spatial skill were gender and school population; consistent with previous literature, females had lower pre-existing spatial skill than males, and online college-age adults as well as high school students from a low socioeconomic status high school had lower spatial skill than students from an academically selective, higher socioeconomic status high school. Thus, we argue that designers of spatial skill training games should target the preferences of female students from non-selective schools, the segment of the population most likely to have low spatial skills. To help game designers target this specific population, we analyze the gaming habits, preferences, and motivations of the low spatial skill subset of our sample and then provide several concrete spatial skill game design recommendations based on our findings.

\section{RELATED WORK}

Our study investigating the relationship between spatial skills and video game play draws on numerous past works studying the relationship between spatial skills and various other factors. We review these past works here and explain how our study builds upon and extends them.

\section{Spatial Skills and Demographics}

One of the most well-known and consistent findings in the research literature on spatial skills is the gender gap. Multiple meta-analyses have found that women and girls score lower than men and boys on various standard tests of dynamic spatial skills such as mental rotation and spatial perception, with effect sizes ranging from 0.44 to 0.90 [35, 40, 42, 74]. These differences have been found to be consistent across age groups [40] and cultures [74], and emerge as early as elementary school (around age 9) [40] or even in some cases as early as age 4 [37]. Gender differences have also been found to widen with age [74]. To isolate the effects of gaming habits, preferences, and motivations, we control for both gender and age in our present study.

Socioeconomic status (SES) is also related to spatial skill. For instance, Levine et al. found that children ages 7-9 with higher SES performed better on a spatial skills test than those with lower SES. In addition, SES and gender interacted in their study such that for low SES students, there was no gender difference in scores [38]. Another study by Noble et al. found that among first children ages 6-7, low SES populations had lower spatial skill than high SES populations [48]. Verdine et al. found that this high SES advantage is present even as early as age 3 [73]. However, there is not yet any body of research we are aware of studying the relationship between SES and spatial skill in adults. We investigate the relationship between demographic characteristics related to SES and spatial skills by analyzing how membership in each of the three populations we recruit participants from (an academically selective high school with higher SES students, a non-selective high school with lower SES students, and college-age adults recruited online) predicts spatial skill.

\section{Spatial Skills and Video Game Play}

Prior work studying the relationship between spatial skills and video game play has largely focused on the genre of action video games, which are generally characterized by the need to attend and react to multiple moving objects in one's field of view and fast-paced motion [27]. Examples of action games include first-person shooters such as Medal of Honor and Halo, but may also include other related genres such as 
Racing or Fighting. Prior work has consistently found that action video game play is associated with higher spatial skill $[9,15,25,26,30]$. Two recent meta-analyses have estimated the effect size at $\bar{g}=0.55$ [6] and $\bar{r}=0.18-0.26$ [60]. Other studies have focused more on the training effects of these commercial games $[18,23,33,50,62,63,65,68]$ or analyzed new games designed specifically for spatial skill training $[17,45,79,82]$.

However, none of these studies has investigated the relationship between spatial skills and video game play in conjunction with demographics. As far as we are aware, there exists only a single study that has done this. Quaiser-Pohl et al. looked at the relationship between gender, game genre preferences, and spatial skill among secondary school students in Germany (ages 10-20). In addition to completing a paper test of spatial skill, students rated how frequently they played each of 8 different video game genres. Students were then grouped into three latent classes based on their stated genre frequencies: "non-players", "action-and-simulation game players", and "logic-and-skill-training players." Quaiser-Pohl et al. found that male action-and-simulation players had higher spatial skill test scores than male non-players, but there was no difference in spatial skill between different player classes for females [56]. More research of this kind, which examines multiple predictors of spatial skill, is needed in order to develop a more complete, up-to-date picture of low spatial skill populations.

\section{Putting It All Together and Adding More}

The present study extends previous work on the relationship between demographics, video game play, and spatial skill in several ways. First, we combine predictors of spatial skill from several different studies: video gameplay habits, genre preferences, gender, age, and SES, in order to build a more complex model of spatial skill predictors and provide a more specific picture of the low spatial skill population. Second, we include participants from three distinct populations: online college-age adults, students from a non-selective, lower SES status high school, and students from an academically selective, higher SES status high school. This diversity of sampling allows our findings to be more generalizable than studies utilizing only a single population. Third, we analyze a set of predictive variables that has not yet been studied: motivations and emotions in gaming. These are important aspects of player experience [7, 11, 36, 43, 61] that can help us build a more in-depth model of low spatial skill populations and understand not just what, but why certain genres or patterns of play might appeal to them. Understanding this specific population is important for designing spatial skill training games because they stand the most to benefit from them. Such games could offer low spatial skill students a fun way to increase their spatial skills to the "threshold" of ability necessary to succeed in early STEM coursework, prevent dropout in STEM majors, and reduce the gender gap in STEM [70, 71].

\section{RESEARCH QUESTIONS}

Our analysis was guided by the following four questions:

RQ1: What video gameplay habits and preferences predict spatial skill independent of gender, age, and population?

RQ2: What motivations for playing video games predict spatial skill independent of gender, age, and population?

RQ3: What emotional gratifications in video games predict spatial skill independent of gender, age, and population?

RQ4: What are the specific gaming habits and preferences of those with the lowest levels of spatial skill?

\section{METHODS}

We conducted an online and in-school study to assess the relationship between pre-existing spatial ability and gaming preferences. The study consisted of a timed test of spatial skill followed by a series of questionnaires asking about participants' gaming habits and preferences.

\section{Recruitment}

We recruited three different populations in the age range 12-22. The first population was a non-selective high school serving primarily low SES students; about $63 \%$ of students are eligible to receive free or reduced lunch, or other low income family services. Our second population was an academically selective high school serving primarily higher income families (only about $9 \%$ of students are eligible for free or reduced lunch). Our third population consisted of college-age adults (ages 18-22) recruited from a large public university, a community college, and various online sources. These three populations were selected to obtain a sample in our target age range that was as diverse as possible. We chose to conduct our study with this age range to strike a balance between a younger population with more time to benefit from spatial skill training and our desire to build upon previous findings in the spatial skill literature, which focuses almost exclusively on college-age adults.

For the college-age population, flyers were posted around campus at a large Midwestern university and a community college, both in the same town, as well as at libraries and coffee shops around the town. Online advertisements were posted on Facebook, Reddit's r/SampleSize subreddit, and in campus email newsletter, and participants could take the survey online at any time and anywhere that they had internet access. At the two high schools, the survey was incorporated into the school day as a class activity that students could participate in with parental permission and consent forms. 
There was no monetary compensation for completing the survey. Instead, we offered a different kind of reward to participants designed to motivate them to take both the test of spatial skill and the questions about their gaming preferences seriously: an opportunity to find out what their primary motivations for gaming were and how well they performed on the spatial test compared to average U.S. adult performance. This form of reward has been used successfully on LabInTheWild.org to attract a large, diverse array of people to participate in online psychology experiments [58].

\section{Survey Procedure}

Upon beginning the survey, participants were asked to read and electronically sign either an assent form (for high schoolers) or a consent form (for online participants). The form explained the purpose of the survey and that participants would receive a summary of their performance on the test and their motivations for gaming at the end. Once participants gave their assent or consent, they began a short timed test of spatial skill: an online version of the redrawn Vandenberg and Kuse Mental Rotations Test (MRT-A) [53].

The MRT is one of the most commonly used assessments of spatial ability [23, 56, 57, 62] and has the advantage of being short, making it feasible for an online study. It consists of two blocks of 12 multiple choice questions. Three minutes are given to complete each block, with a break of two minutes in between (our online version also allowed participants to continue to the next block before the two minutes were up if they wanted). For each question, participants must select from the available answers which two represent the exact same object as a given exemplar figure (see Figure 1). The MRT includes a set of written instructions and four practice problems with correct answers provided to ensure participants understand the task before they start, which we reproduced in the online version. In between survey administration at the high schools and online deployment, we implemented logging of time spent on the test as a way of checking whether participants took the test seriously.

After finishing the test, participants were asked about their gaming habits and preferences. The first survey question asked if the participant had ever played video games. If they had, they were asked a series of follow-up questions related to how recently and how often they played video games (for how many years, hours per week, length of play session), then asked to name their 3 favorite genres and their top 3 favorite games (digital or non-digital). If the participant indicated they had never played video games, they were asked the same questions about games in general, and the questions about recent play, years of play, and favorite video game genres were omitted.

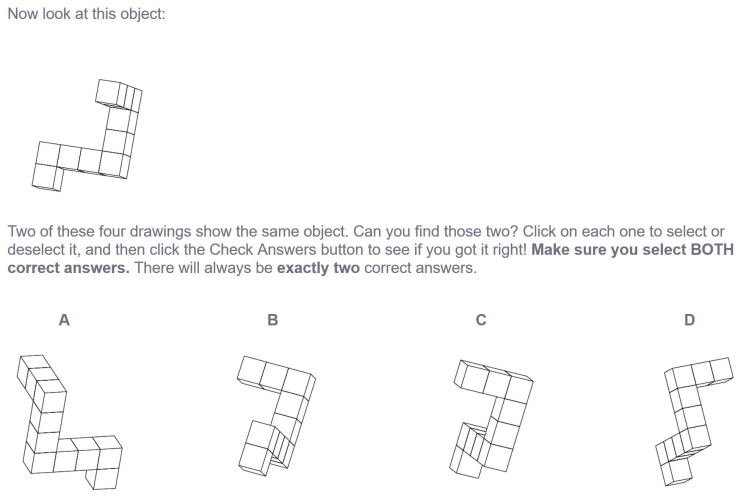

Figure 1: A practice question from the online MRT test. An exemplar figure is shown, and test takers must choose which 2 of the 4 drawings below correspond to an identical figure.

Next, all participants completed the Digital Games Motivation Scale (DGMS), an internationally validated questionnaire used to assess different motivations for playing games [20,21], and an Emotional Gratifications questionnaire to assess participants' most valued emotional experiences in games (see Table 1). Developed by Bartsch [5], it was originally designed for movie and television experiences, but it has been adapted previously by other researchers for video games [10] - we use an adapted version similar to theirs. We included these questionnaires to provide insight into the "why" behind participants' gaming habits and genre preferences. We added an attention check question to both questionnaires, which asked the participant to select a specific answer choice. Those who failed to answer with the requested choice for either questionnaire would be marked as failing the attention check.

Finally, participants completed an optional demographic survey, which asked them to state their gender, age, and country of residence. We also asked participants if they had completed this survey already and provided a text box for them to mention any technical difficulties they had encountered on the spatial skill test or surveys. Once this section was complete, participants saw a summary page describing their performance on the spatial skill test relative to the United States adult average, as well as bar graphs showing their strongest motivations for playing games and their most valued emotional experiences in games, which corresponded to participants' scores on each construct in the DGMS and Emotional Gratifications questionnaires, respectively.

\section{Data Preparation}

In total, we gathered data from 506 participants (235 from the selective high school, 63 from the non-selective high school, and 208 from the online survey for adults). 
For the public selective and non-selective high school samples, we removed the data of participants who failed attention checks on the DGMS or Emotional Gratifications questionnaire or did not complete the entire survey (selective: $n=14$, non-selective: $n=22$ ) as well as those who reported having technical problems during the spatial skill test (selective: $n=1$, non-selective: 0 ). This left us with 220 and 41 participants for the selective and non-selective high schools, respectively.

For the online sample, we removed the data of participants who were not between the ages of 18 and $22(n=73)$, followed by those who failed to input a valid age $(n=23)$, those who indicated that this was not their first time taking our survey $(n=9)$, those who failed attention checks on the questionnaires or did not complete the entire survey $(n=8)$, and those who reported technical problems $(n=7)$. Two additional participants took less than 30 seconds to complete each section of the test (less than 3 seconds per question) and scored 5 out of 24 possible points, below the level of chance. This indicated that these participants did not take the test seriously, and thus we omitted their data as well, leaving us with a final count of 89 online participants.

\section{Data Summary}

Our cleaned sample consisted of 350 participants. In the selective high school sample, $48 \%$ (106) identified as female, $50 \%$ (111) as male, and 2\% (3) as a different gender, of whom one specified their gender as Genderfluid. In the non-selective high school sample, 41\% (17) students identified as female, $56 \%$ as male, and the remaining student as Transgender Male. The age range of selective high school students was 12-17, while for the non-selective high school it was 14-22 (6 chose not to answer, only one student reported an age higher than 18). All but two participants in the online sample reported their country of residence as the United States (98\%). The remaining two were from Singapore and South Korea, respectively. In addition, $2 \%$ of the selective high school students (5) and $7 \%$ of the online sample (6) reported never having played video games. All of the non-selective high school students reported playing video games. Cronbach's $\alpha$ for each construct on the DGMS and Emotional Gratifications questionnaire ranged from 0.73-0.91, indicating good reliability.

\section{Grouping Games \& Game Genres}

The 15 video game genre list used in our study is adapted from Wauck et al's [78] and includes the following genres: Role-Playing Game (RPG), Action, First Person Shooter (FPS), Strategy, Adventure, Simulation, Music, Fighting, Family, Racing, Fitness, Sports, Platformer, Puzzle, and Other. The Action genre included in our 15 genre list is intended to be a catch-all category for games that people generally consider to be action games but that do not fall into any of the other action-related categories (e.g. arcade games).
However, prior work analyzing the relationship between spatial skills and video game genre preferences generally categorizes games and game genres into a more manageable number of categories. Usually, there are two: "Action" or "Non-Action" [6, 9, 15, 25, 26, 54, 60]. However, there is substantial disagreement about what defines an action game [49]. Therefore, we categorized our participants' favorite genres as "Action" or "Non-Action" first using a more broad set of criteria and then using a more restrictive set.

Looking at the academic research literature on spatial skills and action gameplay, we found that some studies equated first person shooters with action games [6, 23, 54], while others named some exemplar action games [9, 15, 27] with genres corresponding to the following genres in our original 15 item list: Action, Platformer, First Person Shooter, Sports, Simulation, Fighting, and Racing. In the industry sphere, Ernest Adams' Fundamentals of Game Design reference book mentions a few subgenres of the Action game genre, two of which correspond to genres in our original list: Fighting and Platformer [3]. A list from a recent LifeWire article includes the subgenres Shooter and Platformer [52]. TvTropes' action subgenres of Platformer, Fighting, and First-Person Shooter [2] are found on our 15 genre list, as are BoardGameGeek's subgenres of Fighting and Platformer [1].

Taking the disjunction of all of these definitions as our broad definition of action games, we ended up with the following list of "Action" genres: Action, Platformer, FirstPerson Shooter, Sports, Simulation, Fighting, and Racing. However, only a single game in Castel et al's list of "Action" games was tagged as Simulation: NHL 2002. Since this game was also tagged as Sports, we decided to remove Simulation from our list, leaving us with Action, Platformer, First-Person Shooter, Sports, Fighting, and Racing. If a participant's list of favorite genres included any genre from our action genre list, we set the variable Action Favorite Genre (Broad) to 1 (true), and if not, 0 (false). For our strict definition of Action genre (Action Favorite Genre (Restrictive)), we took the conjunction of all definitions from the literature, leaving us with the First-Person Shooter (and the Action genre by definition).

Another approach to grouping video game genres is latent class analysis, as in Quaiser-Pohl et al. [56]. Using this approach, we clustered our video game-playing participants' favorite genres from our 15 item list with the $\mathrm{R}$ package poLCA using 2-7 class solutions. Each solution was run 100 times with a maximum number of 5000 iterations. Due to the large number of parameters (participants could select up to 3 favorite genres), we used the Akaike Information Criteria (AIC) (and interpretability) to evaluate each solution. The four class solution provided the best balance between low AIC and interpretability. We interpreted the four classes as "Action Gamers" (favoring Action, FPS, Fighting, 
and Other), "Cognitive Gamers" (favoring Strategy and Puzzle), "Role-Playing Gamers" (favoring RPG and Simulation), and "Sports/Social Gamers" (favoring Racing, Fitness, Sports, and Family). We assigned each participant their predicted genre class as the variable Favorite Genre Class.

To categorize participants' favorite games as Action or Non-Action, we simply used genre tags from the reviewaggregation site Metacritic. If a game's tags included the word "Action" (e.g., "Action", "Action Adventure", "Action RPG"), we counted the game as Action; if not or if the listed game was not a video game, we counted it as Non-Action. If any of a participant's favorite games was an action game according to this definition, we assigned the participant a value of 1 (true) for the Action Favorite Game variable, and 0 (false) if otherwise. If the game was a video game but not listed on Metacritic (e.g. "Brawl Stars"), or its description was too vague to uniquely identify it (e.g., "Mario", "Telltale Games"), we marked it as "neither" and looked at the rest of the games the participant listed. If no other games were categorized as Action (meaning that this game would be the deciding factor in whether Action Favorite Game was 1 or 0 ), we omitted the participant's data from our dataset $(n=14)$.

\section{RESULTS}

The analysis of our data proceeded in two stages. First, to understand what gaming preferences predicted spatial skill, we conducted hierarchical regressions analyzing the relationship between demographics, gaming habits and preferences, and spatial skills. Next, we used the results of our regression analysis to identify the subset of our sample with the lowest spatial skills and characterize their gaming habits and preferences in order to develop a set of recommendations for designers of spatial skill training games.

\section{Hierarchical Regression Analysis}

Our regression analysis consisted of 3 hierarchical regressions with our entire sample of video game players $(n=350)$. According to the criteria used by Wilson Van Voorhis et al., this sample size provides sufficient statistical power for the number of variables we are analyzing [81]. We chose a hierarchical analysis because we were interested primarily in gaming preferences as predictors of spatial skill after taking into consideration demographic variables' predictive power. For each regression, participants' score on the spatial skill test was the dependent variable. We entered the following demographic variables in the first block: gender (only male and female were used due to the small number of participants (5) identifying as a different gender), age, and population (selective high school, non-selective high school, online). We entered the following gaming experience variables in the second block (See Table 1 for details):
Table 1: Hierarchical Regression Measures. *See Grouping Games \& Game Genres section. NSHS = nonselective high school, SHS = selective high school.

\begin{tabular}{|c|c|}
\hline Measure & Scale \\
\hline Gender & $\mathrm{F} / \mathrm{M}^{*}$ \\
\hline Age & Number \\
\hline Population & NSHS/SHS/Online \\
\hline Played Videogames Recently & $\mathrm{Y} / \mathrm{N}$ \\
\hline How Long Played Videogames & $1-5(<6$ mo. $-10+$ yrs $)$ \\
\hline Weekly Hours & Number \\
\hline Session Duration & $1-5(<15$ min. $-4+$ hrs $)$ \\
\hline Action Fav. Game & $\mathrm{Y} / \mathrm{N}^{*}$ \\
\hline Action Fav. Genre (Broad) & $\mathrm{Y} / \mathrm{N}^{*}$ \\
\hline Action Fav. Genre (Restrictive) & $\mathrm{Y} / \mathrm{N}^{*}$ \\
\hline Fav. Genre Class & $\mathrm{Y} / \mathrm{N}^{*}$ \\
\hline Habit & 1-5 (Disagree-Agree) \\
\hline Moral Self-Reaction & 1-5 (Disagree-Agree) \\
\hline Agency & 1-5 (Disagree-Agree) \\
\hline Narrative & 1-5 (Not-Very Important) \\
\hline Escapism & 1-5 (Not-Very Important) \\
\hline Pastime & 1-5 (Not-Very Important) \\
\hline Performance & 1-5 (Not-Very Important) \\
\hline Social & 1-5 (Not-Very Important) \\
\hline Contemplative Experiences & 1-5 (Disagree-Agree) \\
\hline Fun & 1-5 (Disagree-Agree) \\
\hline Thrill & 1-5 (Disagree-Agree) \\
\hline Character Engagement & 1-5 (Disagree-Agree) \\
\hline Vicarious Release of Emotions & 1-5 (Disagree-Agree) \\
\hline Empathic Sadness & 1-5 (Disagree-Agree) \\
\hline Social Sharing of Emotions & 1-5 (Disagree-Agree) \\
\hline
\end{tabular}

(1) Habits Regression: Played Videogames Recently, How Long Played Videogames, Weekly Hours, Session Duration, Action Favorite Game, Action Favorite Genre (Broad), Action Favorite Genre (Restrictive), and Favorite Genre Class.

(2) Motivations Regression: Habit, Moral Self-Reaction, Agency, Narrative, Escapism, Pastime, Performance, and Social constructs from the DGMS [20].

(3) Emotional Gratifications Regression: Contemplative Experiences, Fun, Thrill, Character Engagement, Vicarious Release of Emotions, Empathic Sadness, and Social Sharing of Emotions constructs from the Emotional Gratifications questionnaire [5].

All regressions were performed using the statistical software package R. For each regression described below, diagnostic plots indicated that the assumptions of linearity of 
the data, normality of residuals, homoscedasticity, and independence of observations were met, and no variables had variance inflation factors greater than 2 .

Video Game Players Only. Our first regression model, Habits, looked at predictors of spatial skill related to participants' gameplay habits and genre preferences. Before running the model, we noticed that two participants had given extremely high answers for the Weekly Hours question: 90 and 100 hours, so we omitted these participants' data from our Habits model. With only the first block added, the model was significant $(F(4,259)=12.51, p<0.0001)$ and explained $15 \%$ of the variance in the data (adjusted $R^{2}=0.15$ ). Only male gender $(\beta=3.43, t=5.57, p<0.0001)$ was a significant predictor of spatial skill. In the second block of habits variables, Action Favorite Game (Broad) was the only significant predictor ( $\beta=1.81, t=2.07, p=0.040)$, but the second block did not significantly improve the model $(F(9,250)=1.24, p=0.27)$.

There were a significant number of missing Weekly Hours responses $(n=52)$, especially from the non-selective high school, which caused a large amount of our data to be omitted from the Habits model. Therefore, we reran the Habits model again but with the Weekly Hours variable omitted. In the new Habits model, the first block was significant $(F(4,301)=16.46, p<0.0001)$ and explained $17 \%$ of the data's variance. Male gender $(\beta=3.56, t=6.25, p<0.0001)$ and being a selective high school student $(\beta=2.52, t=2.03$, $p=0.043)$ were both predictors of spatial skill. Adding the second block of habits variables did not significantly improve the model $(F(8,293)=1.34, p=0.22)$.

Our second model, Motivations for Playing, also had a significant first block $(F(4,321)=18.00, p<0.0001)$ explaining $17 \%$ of the variance, with male gender $(\beta=3.52, t=6.58$, $p<0.0001)$ and selective school population $(\beta=2.45$, $t=2.075, p=0.039$ ) as the only predictors of spatial skill. Adding the second block of motivation constructs from the DGMS did not reveal any significant predictors, explained only $1 \%$ of additional variance, and did not improve the model significantly $(F(8,313)=1.41, p=0.19)$.

Our third and final model, Emotional Gratifications for Playing, revealed the same pattern of results: significant first block $(F(4,321)=18.00, p<0.0001)$ explaining $17 \%$ of the variance, with male gender $(\beta=3.53, t=6.58, p<0.0001)$ and membership in the public selective school population ( $\beta=2.45, t=2.08, p=0.039$ ) as the only predictors of spatial skill. Adding the second block of emotion constructs explained only $2 \%$ of additional variance and did not improve the model significantly $(F(7,314)=1.98, p=0.057)$.

In summary, our models showed consistently that among participants who played video games, no gaming habits or preferences (RQ1), no motivations for gaming (RQ2), and

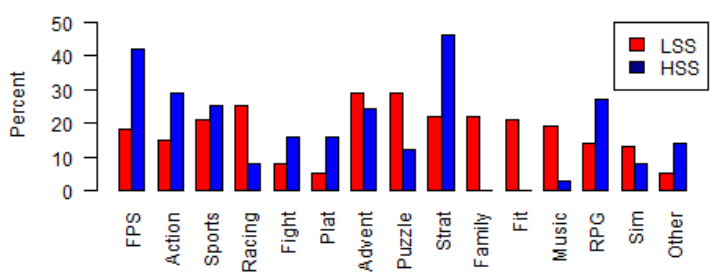

Figure 2: Video game genre preferences for the LSS group, as compared to the HSS group. FPS $=$ First Person Shooter, RPG = Role-Playing Game. Percentages do not add up to 100 because participants could choose up to 3 favorite genres.

no emotional gratifications (RQ3) improved the model's predictive power over and above what the first block of demographic factors provided. Only gender and population were predictive of spatial skill.

Adding in Non-Video Game Players. While participants who reported never playing video games constituted a relatively small portion of our sample (11 participants, 3\%), excluding them might bias our data more in favor of action video gamers, since non-video game players by definition do not play action video games. For this reason, we decided to redo the above analyses to include non-video game players. In order to do this, we had to remove the variables Played Videogames Recently and How Long Played Videogames from the Habits model since they were questions about video gameplay habits and thus not applicable to non-video game players. In addition, we assigned a value of 0 (false) to the Action Favorite Game and Action Favorite Genre variables for each non-video game player in the sample.

Rerunning the Habits, Motivations, and Emotions regression models, we found that the Habits model stayed mostly the same. However, the first block (gender, age, and population) explained more of the variance (18\%), and the second block of the Motivations for Playing model became a significant improvement over the first block $(F(8,324)=2.05$, $p=0.040)$, explaining an additional $2 \%$ of the variance. Within the second block, habit was the only DGMS construct associated with spatial skill ( $\beta=0.27, t=2.63, p=0.009)$, and the association had little practical importance given its low beta value; increasing average habit score from 1 , the minimum possible, to 5 , the maximum possible, would only add about one point to the predicted spatial skills test score. The Emotional Gratifications model did not change significantly (gender and population were the only predictors, and the second block did not improve the model). All in all, adding non-video game players to our models did not change them in any significant way. 


\section{Low Spatial Skill Population Preferences}

Taken together, our regression models suggest that the only predictors of spatial skill across our three study populations were gender and population, with male participants and students at the selective high school scoring higher on the spatial skills test than females and participants from the other two populations. Male gender and being a student at the selective high school each add about 2.5-3.5 points to one's spatial skill test score, meaning that a male selective high school student is predicted to score 6 points higher (out of 24 possible points) than a female participant from the online population or the non-selective high school.

Thus, our data suggests that female participants from the online or non-selective high school populations constitute the lowest spatial skill group in our sample. To provide insight into what this demographic looks for in a game, we analyzed their gaming habits, genre preferences, motivations, and emotional gratifications. Henceforth, for the sake of brevity, we will refer to the subset of our participants who are female and come from the online or non-selective high school as the low spatial skills (LSS) group $(n=85)$.

The LSS group reported playing games most often in fairly short sessions; their most popular answer choice was "1559 minutes" (43\%), and about equal numbers of them chose "Less than 15 minutes" (22\%) and "1-2 hours" (24\%). Reported weekly hours of gameplay tended to be somewhat low in the LSS group. Those in the LSS group who answered the question about weekly gameplay hours $(69 \%)$ reported a mean of 2.95 hours a week (median $=2, \min =0, \max =20$ ).

Figure 2 summarizes the LSS group's favorite video game genres compared to the preferences of the participant group with the highest spatial skill (male selective high school students, HSS). Six LSS participants did not provide favorite video game genres because they reported not playing video games. The two most popular genres in the LSS group were Adventure and Puzzle, which were each chosen by $32 \%$ of the group. While $67 \%$ of the LSS group's video game players chose favorite genres that fell into our broadly-defined "Action" genre (Action, Platformer, First-Person Shooter, Sports, Fighting, and Racing), Figure 2 shows that most of this "Action" preference is due to a preference for the Racing and Sports genres. Genres in our more restrictive "Action" game grouping (including only the First-Person Shooter and Action categories) were much more popular with the HSS group (chosen by $42 \%$ and $29 \%$, respectively) than with the LSS group (chosen by $19 \%$ and $16 \%$, respectively).

We looked up the Metacritic genre tags for each participant's favorite video games in order to understand participants' genre preferences in more detail. LSS group members listed 101 favorite video games in total, which generated 79 unique tags. Each game had 2-6 tags. Although the Miscellaneous and General tags occurred very frequently, we chose to ignore them as they were not descriptive and always occurred in the presence of more descriptive genre tags. Eight LSS participants (9\%) did not list any favorite games.

Analysis of LSS participants' favorite games revealed a pattern not evident in the favorite genre data: while only a little over half of the LSS group (59\%) listed at least one video game, Action game preferences were strong among those who did. The four most popular video game genre tags were all highly related to both our more broad and more restrictive definitions of "Action" genre: Action, Action-Adventure, First-Person, and Shooter. Among those in the LSS group who listed at least one video game $(n=45), 67 \%$ named a game tagged as Action, and the tags Action-Adventure, FirstPerson, and Shooter were each named by $29 \%$ of them. In total, $80 \%$ of those who listed at least one video game named at least one favorite game with a tag including the word "Action" (Action, Action-Adventure, and Action-RPG). In addition, only $16 \%$ of the LSS group listed both video games and non-video games as favorites, suggesting that there may be two distinct groups of game type preferences among the LSS group: digital and non-digital.

Overall, the LSS group indicated that they were moderately motivated by most of the DGMS constructs. They generally felt somewhat positive about spending time playing games, scoring a median of 3.67 on the Moral Self-Reaction construct. In addition, they were moderately motivated by the desire to perform and achieve (Performance, median = 3.33), a sense of agency (Agency, median = 3), the in-game narrative (Narrative, median $=3$ ), playing just to pass the time (Pastime, median $=3$ ), and the desire to escape from daily life (Escapism, median $=2.67)$. LSS participants were less motivated by getting to interact with other players (Social, median $=2.33$ ), and playing out of habit (Habit, median $=2$ ), each with a particularly low mode of 1 . Thus, most LSS participants have a diverse array of motivations for playing games, but may not be particularly regular or social gamers.

The Emotional Gratifications questionnaire revealed that the LSS group valued mainly pleasurable, hedonistic emotional experiences in games; the only constructs on which they scored higher than 3 (Neither Agree Nor Disagree) were Fun (median=3.75) and Thrill (median=3.5). LSS group members scored a median of 2-3 on the remaining constructs of Character Engagement, Social Sharing of Emotions, Contemplative Experiences, Vicarious Release of Emotions, and Empathic Sadness. While the distribution of Character Engagement scores seemed to be somewhat bimodal with peaks at 1 and 4, suggesting two distinct camps of pro- and anticharacter engagement, scores on the remaining constructs were consistently low, indicating that the LSS group did not value these more neutral to negative emotional experiences 
very much in gameplay. This was especially true of Empathic Sadness, which had a mode of 1 .

Taken together, our findings regarding the gaming habits, preferences, and motivations of LSS participants (RQ4) suggest that this subgroup is split fairly evenly between a preference for digital games and a preference for non-digital games. Those whose favorite games are video games tend to favor certain subgenres of Action video games as well as the non-Action genres, but the LSS group in general seems to enjoy the Adventure and Puzzle genres the most. Overall, LSS participants prefer short play sessions, have many different motivations for gaming, and value the emotions of fun and thrill the most in gaming experiences, but do not generally play habitually or socially and do not value more negative emotional experiences in games.

\section{DESIGN RECOMMENDATIONS}

Focusing on the low spatial skill (LSS) group in our sample and analyzing their gaming habits, preferences, and underlying motivations for gaming allowed us to obtain a more detailed picture of the kinds of games they might be the most interested in. Here, we summarize this picture and provide several recommendations to designers of game-based spatial skill training interventions based on our findings.

Facilitate Short Gameplay Sessions. Since the LSS group reported playing in short sessions (about 15-59 minutes), reported a low number of hours played per week, and were motivated to play games in part by the desire to just pass the time, we recommend that game interventions focus on providing a gameplay experience that is easy to engage and disengage with to facilitate short sessions. One way to do this might be to make game levels completable in less than 15 minutes each, allowing players to feel a sense of accomplishment and progression despite short play sessions. Mobile games are particularly well-suited to short sessions [8].

Promote Simple Fun and Thrill. LSS participants' responses to the Emotional Gratifications questionnaire indicated that they valued hedonistic emotional experiences in games and put less value in emotional experiences that were more social and cognitive in nature or more negative. Game designers can accommodate these emotional gratifications by designing games to emphasize the more immediate pleasures of gameplay - simple fun, of course, but also thrill. Thrill can be elicited in gameplay by creating tense, suspenseful situations, such as the pressure to overcome a challenge within a certain time limit or complete a mission objective without being discovered and attacked by enemy forces.

Get Creative With Adventure and Puzzle Genres. The LSS group's gaming preferences seemed to be split along a digital divide: our analysis of their favorite games revealed that a large portion of them seemed to prefer action video games, while many others preferred non-digital games, as evidenced by their responses to the question about favorite games. How can game designers reconcile these two sets of preferences in practice? Here, our findings regarding the LSS group's video game genre preferences may provide insights. Overall, the most popular genres with the LSS group were Adventure and Puzzle, which may indicate some common ground between digital and non-digital gamers' preferences.

Game designers may therefore want to focus on these two genres when designing spatial skill training video games, especially since they lend themselves well to being combined with other genres - like the action games many LSS gamers enjoy. For instance, fast-paced first-person shooter gameplay could be combined with an overarching story, as is done in many Action-Adventure games, and would also support one of the LSS group's stronger gameplay motivations: Narrative. To accommodate non-digital players, Adventure and Puzzle games could be adapted to non-digital formats. Text-based adventures could be designed with spatial features (e.g., having to navigate through buildings or caves and gradually build a mental map of the area as features are described to the player), and many board games exist already that present puzzle-esque spatial challenges, such as the laying out of complex tunnel pathways in the board game Saboteur or the spatial planning required for moves in checkers and chess.

Another advantage of focusing on Adventure and Puzzle genres is that they may be easier to combine with spatiallyrelevant features. While very little is known empirically about which game features are spatially relevant, some preliminary steps in this direction have been taken by Wauck et al. and Xiao et al., who found that performance on first person exploration and 3D object construction tasks within a computer game correlated with spatial skill [77, 82]. In addition, Chang et al. and Mazalek et al. found that a first person exploration VR game with tangibles improved players' spatial skills in the short term [17, 45]. Each of these in-game tasks map well to the Adventure and Puzzle genres and demonstrate how a synergy between spatially-relevant features and LSS population preferences might be achieved. However, these studies are preliminary work with underpowered samples, so instead of or in addition to the feature sets they recommend, game designers may want to try incorporating features found in games shown empirically to train spatial skills, such as Medal of Honor (Action, First-Person Shooter), Portal 2 (Action, First-Person Shooter, Puzzle), or Super Mario 64 (Action, Platformer).

\section{DISCUSSION}

In this study, we investigated four research questions, RQ1, RQ2, RQ3 and RQ4, regarding the gaming habits, preferences, and motivations of low spatial skill teens and young 
adults in order to provide player-centered design guidance for game-based spatial skill training interventions that aim to increase students' efficacy in STEM majors and careers. Our findings are consistent with prior work showing a male and high socioeconomic status advantage in spatial skill [38, 40, 48, 71, 73], but inconsistent with prior work showing a relationship between action gaming and spatial skill $[6,9,15,25,26,30,60]$.

A likely reason for this failure to replicate is that we analyzed only preference for action games, whereas these previous works all measured actual frequency of action gameplay, which may be more relevant to spatial skills. Our different results may also be due to the fact that nearly all prior work used extreme groups analysis [6, 9, 15, 25, 26, 30], and had very low sample sizes ( $n \leq 20$ per comparison group for individual studies). Extreme groups analysis and low sample size can sometimes lead to overestimated effect sizes, a scenario less likely with our larger sample size and correlation analysis [12, 19, 69]. Finally, our results may have been affected by the over-representation of selective high school students in our sample relative to the other populations, causing our results to be less representative of the general population of 14-22 year olds.

Given our inconclusive results, we advise game designers to not worry too much about whether or not to use action games for training interventions. It is far more important to incorporate the more specific, fine-grained gaming preferences of low spatial skill populations so that the game intervention is actually something they would want to play. By asking more detailed and fine-grained questions about participants' gaming habits, preferences, and motivations, we were able to provide more sophisticated, concrete recommendations than would have been possible with more simplistic measures of gameplay habits and genre preferences that have typically been used in past work $[9,15,25,26,30]$.

Since we found that LSS group members seemed divided between a preference for digital and non-digital games, one might reasonably ask if this group is indeed the best target audience for video game-based spatial skill training interventions. We believe it is; while there was certainly a digital/nondigital split in game preference, the majority (59\%) of the LSS group named a video game as one of their favorite games, indicating that a large chunk of the low spatial skill population could be open to a video game-based intervention. However, we are not suggesting that those with a preference for non-digital games should be ignored; rather, we recommend game designers consider how to apply our design recommendations to both digital and non-digital spatial skill training games. Designing for digital and non-digital interventions allows game designers to target a wider section of the low spatial skill population - those who have the most to gain from spatial skill training interventions that can help them achieve the threshold of spatial ability necessary to succeed in STEM majors and future careers [70, 71].

We set out in this work to guide game designers in a playercentered approach to spatial skill training game design to improve the STEM efficacy of low spatial skill students, but the approach is applicable to the design of any educational or cognitive training game. What is exciting about the possibility of using games as interventions is not simply that the intervention will train a skill, but that people will actually want to do the training, just for the intrinsic fun of it. These same skills, after all, can be trained in laboratory or classroom settings using traditional workbook exercises, but this requires extrinsic compensation, whether in the form of money or course credit; as soon as the extrinsic compensation ends, participants are likely to stop training by themselves. The intrinsic fun offered by game training - if the game is designed with the target population's preferences in mind - offers a way for those who stand the most to benefit from training to obtain these benefits relatively painlessly, utilizing their leisure time for informal learning [34] rather than replacing the precious few moments of leisure they have with something that feels like work.

\section{LIMITATIONS}

The biggest limitation of the present work is that our participants' data about gameplay habits and preferences was based entirely on self-report measures, which can often differ significantly from their actual behavior [47, 80]. However, even if participants' expressed desire of what is important to them in a game is inaccurate, it is still valuable for the purpose of designing a game to appeal to them because it can still tell us what people may look for first when choosing a new game to play. Another limitation of this study was that our higher socioeconomic status, selective high school population was overrepresented in the sample compared to the other two populations. We plan to address this limitation in future work by establishing research relationships with a wider range of schools, particularly those in lower income communities. A final limitation of this work is that despite our findings that gender and source population predicted spatial skill, our models only explained $15 \%-19 \%$ of the variance in participants' spatial skills. Future work could introduce more potentially relevant variables, such as frequency and type of spatial non-video game activities $[32,73]$.

\section{CONCLUSION}

In this paper, we have presented what is to date the most comprehensive investigation of the relationship between preexisting spatial skill and video game play habits, preferences, and underlying motivations, making use of a combination of online and in-school sources to increase the diversity of our subject pool. Our investigation took a player-centered 
approach, focusing on identifying the subset of the teen and college age population with the lowest spatial skill, who have the most to gain from spatial skill training interventions aimed at increasing STEM readiness: females from a less socioeconomically advantaged background. We then analyzed the gaming habits, preferences, and motivations of this population in order to provide a set of design recommendations for spatial skill training games. However, our work has broader implications for educational and cognitive training games more generally. By tailoring the design of games to the interests of the subpopulation most likely to benefit from them, game designers and researchers can take advantage of not only the cognitive, but also the motivational benefits of video games - the entire point of using them as learning interventions in the first place.

\section{ACKNOWLEDGMENTS}

The authors would like to thank Jamie Lee for assistance with survey design and India Owens for assistance with proctoring. This material is based upon work supported by the National Science Foundation Graduate Research Fellowship Program under Grant No. DGE - 1144245.

\section{REFERENCES}

[1] 2018. BoardGameGeek: Video Game Guide to Genres. https://boardgamegeek.com/wiki/page/ Video\{_\}Game\{_\}Guide\{_\}to\{_\}Genres

[2] 2018. TvTropes: Action Game. https://tvtropes.org/pmwiki/pmwiki. php/Main/ActionGame

[3] Ernest Adams. 2014. Fundamentals of Game Design (3rd ed.). New Riders Publishing. https://dl.acm.org/citation.cfm?id=2544002

[4] Ian Arawjo, Cheng-Yao Wang, Andrew C. Myers, Erik Andersen, and François Guimbretière. 2017. Teaching Programming with Gamified Semantics. In Proceedings of the 2017 CHI Conference on Human Factors in Computing Systems - CHI '17. ACM Press, New York, New York, USA, 4911-4923. https://doi.org/10.1145/3025453.3025711

[5] Anne Bartsch. 2012. Emotional Gratification in Entertainment Experience. Why Viewers of Movies and Television Series Find it Rewarding to Experience Emotions. Media Psychology 15, 3 (jul 2012), 267-302. https://doi.org/10.1080/15213269.2012.693811

[6] Benoit Bediou, Deanne M Adams, Richard E Mayer, Elizabeth Tipton, C Shawn Green, and Daphne Bavelier. 2017. Meta-Analysis of Action Video Game Impact on Perceptual, Attentional, and Cognitive Skills. Psychological Bulletin (2017). https://doi.org/10.1037/bul0000130

[7] Max V. Birk, Dereck Toker, Regan L. Mandryk, and Cristina Conati. 2015. Modeling Motivation in a Social Network Game Using PlayerCentric Traits and Personality Traits. Springer International Publishing, 18-30. https://doi.org/10.1007/978-3-319-20267-9_2

[8] Matthias Böhmer, Brent Hecht, Johannes Schöning, Antonio Krüger, and Gernot Bauer. 2011. Falling asleep with Angry Birds, Facebook and Kindle. In Proceedings of the 13th International Conference on Human Computer Interaction with Mobile Devices and Services - MobileHCI '11. ACM Press, New York, New York, USA, 47. https://doi.org/10.1145/ 2037373.2037383

[9] Walter R. Boot, Arthur F. Kramer, Daniel J. Simons, Monica Fabiani, and Gabriele Gratton. 2008. The effects of video game playing on attention, memory, and executive control. Acta Psychologica 129, 3 (nov 2008), 387-398. https://doi.org/10.1016/j.actpsy.2008.09.005

[10] Julia Ayumi Bopp, Elisa D. Mekler, and Klaus Opwis. 2016. Negative Emotion, Positive Experience?: Emotionally Moving Moments in Digital Games. In Proceedings of the 2016 CHI Conference on Human Factors in Computing Systems - CHI '16. ACM Press, New York, New York, USA, 2996-3006. https://doi.org/10.1145/2858036.2858227

[11] Elizabeth A. Boyle, Thomas M. Connolly, Thomas Hainey, and James M. Boyle. 2012. Engagement in digital entertainment games: A systematic review. Computers in Human Behavior 28, 3 (2012), 771-780. https: //doi.org/10.1016/j.chb.2011.11.020

[12] Katherine S. Button, John P. A. Ioannidis, Claire Mokrysz, Brian A. Nosek, Jonathan Flint, Emma S. J. Robinson, and Marcus R. Munafò. 2013. Power failure: why small sample size undermines the reliability of neuroscience. Nature Reviews Neuroscience 14, 5 (may 2013), 365-376. https://doi.org/10.1038/nrn3475

[13] Alessandro Canossa and Anders Drachen. 2009. Patterns of Play: Play-Personas in User-Centred Game Development. In Proceedings of DiGRA.

[14] Justine Cassell and Henry Jenkins. 1998. Chess For Girls?: Feminism and Computer Games. In From Barbie to Mortal Kombat: Gender and Computer Games, Justine Cassell and Henry Jenkins (Eds.). The MIT Press, Cambridge, MA, Chapter 1, 1-32.

[15] Alan D. Castel, Jay Pratt, and Emily Drummond. 2005. The effects of action video game experience on the time course of inhibition of return and the efficiency of visual search. Acta Psychologica 119, 2 (jun 2005), 217-230. https://doi.org/10.1016/j.actpsy.2005.02.004

[16] Amanda Chaffin, Katelyn Doran, Drew Hicks, and Tiffany Barnes. 2009. Experimental evaluation of teaching recursion in a video game. In Proceedings of the 2009 ACM SIGGRAPH Symposium on Video Games - Sandbox '09. ACM Press, New York, New York, USA, 79. https: //doi.org/10.1145/1581073.1581086

[17] Jack Shen-Kuen Chang, Georgina Yeboah, Alison Doucette, Paul Clifton, Michael Nitsche, Timothy Welsh, and Ali Mazalek. 2017. Evaluating the effect of tangible virtual reality on spatial perspective taking ability. In Proceedings of the 5th Symposium on Spatial User Interaction - SUI '17. ACM Press, New York, New York, USA, 68-77. https://doi.org/10.1145/3131277.3132171

[18] Isabelle D. Cherney, Kyle Bersted, and Joseph Smetter. 2014. Training Spatial Skills in Men and Women. Perceptual and Motor Skills 119, 1 (aug 2014), 82-99. https://doi.org/10.2466/23.25.PMS.119c12z0

[19] Andrew R. A. Conway, Michael J. Kane, Michael F. Bunting, D. Zach Hambrick, Oliver Wilhelm, and Randall W. Engle. 2005. Working memory span tasks: A methodological review and user's guide. Psychonomic Bulletin \& Review 12, 5 (oct 2005), 769-786. https://doi.org/ 10.3758/BF03196772

[20] Frederik De Grove, Johannes Breuer, Vivian Hsueh Hua Chen, Thorsten Quandt, Rabindra Ratan, and Jan Van Looy. 2017. Validating the Digital Games Motivation Scale for Comparative Research Between Countries. Communication Research Reports 34, 1 (jan 2017), 37-47. https://doi.org/10.1080/08824096.2016.1250070

[21] Frederik De Grove, Verolien Cauberghe, and Jan Van Looy. 2016. Development and Validation of an Instrument for Measuring Individual Motives for Playing Digital Games. Media Psychology 19, 1 (jan 2016), 101-125. https://doi.org/10.1080/15213269.2014.902318

[22] Michel Dorval and Michel Pepin. 1986. Effect of Playing a Video Game on a Measure of Spatial Visualization. Perceptual and Motor Skills 62, 1 (feb 1986), 159-162. https://doi.org/10.2466/pms.1986.62.1.159

[23] Jing Feng, Ian Spence, and Jay Pratt. 2007. Playing an Action Video Game Reduces Gender Differences in Spatial Cognition. Psychological Science 18, 10 (oct 2007), 850-855. https://doi.org/10.1111/j.1467-9280. 2007.01990.x 
[24] Isabela Granic, Adam Lobel, and Rutger C. M. E. Engels. [n. d.]. The benefits of playing video games. ([n. d.]).

[25] C.Shawn Green and Daphne Bavelier. 2007. Action-Video-Game Experience Alters the Spatial Resolution of Vision. Psychological Science 18, 1 (jan 2007), 88-94. https://doi.org/10.1111/j.1467-9280.2007.01853.x

[26] C. Shawn Green and Daphne Bavelier. 2003. Action video game modifies visual selective attention. Nature 423, 6939 (may 2003), 534-537. https://doi.org/10.1038/nature01647

[27] C. Shawn Green and Daphne Bavelier. 2006. Effect of action video games on the spatial distribution of visuospatial attention. Fournal of Experimental Psychology: Human Perception and Performance 32, 6 (2006), 1465-1478. https://doi.org/10.1037/0096-1523.32.6.1465

[28] Shawn Green and Daphne Bavelier. 2004. The Cognitive Neuroscience of Video Games. Digital Media: Transformation in Human Communication (2004), 132. http://isites.harvard.edu/fs/docs/icb.topic951141.files/ cognitiveNeuroOfVideoGames-greenBavelier.pdf $\{\%\}$ Cnpapers3: //publication/uuid/254EEDEA-EDB3-431C-B8CF-84C73380D94D

[29] B. S. Greenberg, J. Sherry, K. Lachlan, K. Lucas, and A. Holmstrom. 2010. Orientations to Video Games Among Gender and Age Groups. Simulation \& Gaming 41, 2 (apr 2010), 238-259. https://doi.org/10. $1177 / 1046878108319930$

[30] Patricia M. Greenfield, Craig Brannon, and David Lohr. 1994. Twodimensional representation of movement through three- dimensional space: The role of video game expertise. Journal of Applied Developmental Psychology 15 (1994), 87-103. https://doi.org/10.1016/0193-3973(94) 90007-8

[31] James D. Ivory. 2006. Still a Man's Game: Gender Representation in Online Reviews of Video Games. Mass Communication and Society 9, 1 (feb 2006), 103-114. https://doi.org/10.1207/s15327825mcs0901_6

[32] Jamie J. Jirout and Nora S. Newcombe. 2015. Building Blocks for Developing Spatial Skills: Evidence From a Large, Representative U.S. Sample. Psychological Science 26, 3 (2015). https://doi.org/10.1177/ 0956797614563338

[33] Simone Kühn, Tobias Gleich, Robert C. Lorenz, Ulman Lindenberger, and Jürgen Gallinat. 2014. Playing Super Mario induces structural brain plasticity: gray matter changes resulting from training with a commercial video game. Molecular psychiatry 19, August 2013 (2014), 265-71. http://www.ncbi.nlm.nih.gov/pubmed/24166407

[34] H Chad Lane. 2013. Enhancing informal learning experiences with affect-aware technologies. In Handbook of Affective Computing, R. A Calvo, S. D'Mello, Jonathan Gratch, and A. Kappas (Eds.). Oxford University Press. http://www.move2learn.education.ed.ac.uk/wp-content/ uploads/2015/04/Lane14-EnhancingInformalLearning-FINAL.pdf

[35] Carol A. Lawton. 2010. Gender, Spatial Abilities, and Wayfinding. In Handbook of Gender Research in Psychology. https://doi.org/10.1007/ 978-1-4419-1465-1_16

[36] Nicole Lazzaro. 2009. Why We Play: Affect and the Fun of Games. In Human-Computer Interaction: Designing for Diverse Users and Domains, Andrew Sears and Julie A. Jacko (Eds.). CRC Press, Boca Raton, FL, Chapter 10, 155-175.

[37] Susan C. Levine, Janellen Huttenlocher, Amy Taylor, and Adela Langrock. 1999. Early sex differences in spatial skill. Developmental psychology 35, 4 (1999), 940-949.

[38] Susan C. Levine, Marina Vasilyeva, Stella F. Lourenco, Nora S. Newcombe, and Janellen Huttenlocher. 2005. Socioeconomic status modifies the sex difference in spatial skill. Psychological Science 16, 11 (nov 2005), 841-845.

[39] Lynn S. Liben. 2015. The STEM Gender Gap: The Case for Spatial Interventions. International fournal of Gender, Science and Technology 7, 2 (2015), 133-150.
[40] Marcia C. Linn and Anne C. Petersen. 1985. Emergence and Characterization of Sex Differences in Spatial Ability: A Meta-Analysis. Child Development 56, 6 (dec 1985), 1479. https://doi.org/10.2307/1130467

[41] Derek Lomas, Kishan Patel, Jodi L. Forlizzi, and Kenneth R. Koedinger. 2013. Optimizing challenge in an educational game using large-scale design experiments. In Proceedings of the SIGCHI Conference on Human Factors in Computing Systems - CHI '13. ACM Press, New York, New York, USA, 89. https://doi.org/10.1145/2470654.2470668

[42] Yukiko Maeda and So Yoon Yoon. 2013. A Meta-Analysis on Gender Differences in Mental Rotation Ability Measured by the Purdue Spatial Visualization Tests: Visualization of Rotations (PSVT:R). Educational Psychology Review 25, 1 (2013), 69-94. https://doi.org/10. 1007/s10648-012-9215-x

[43] Thomas W. Malone and Mark R. Lepper. 1987. Making Learning Fun: A Taxonomy of Intrinsic Learning. Aptitude, learning, and instruction 3 (1987), 223-253. http://ocw.metu.edu.tr/mod/resource/view.php?id= 1311

[44] Taylor Martin, Carmen Petrick Smith, Nicole Forsgren, Ani Aghababyan, Philip Janisiewicz, and Stephanie Baker. 2015. Learning Fractions by Splitting: Using Learning Analytics to Illuminate the Development of Mathematical Understanding. Fournal of the Learning Sciences 24, 4 (oct 2015), 593-637. https://doi.org/10.1080/10508406. 2015.1078244

[45] Ali Mazalek, Sanjay Chandrasekharan, Michael Nitsche, Tim Welsh, Paul Clifton, Andrew Quitmeyer, Firaz Peer, Friedrich Kirschner, and Dilip Athreya. 2011. I'm in the game: embodied puppet interface improves avatar control. In Proceedings of the fifth international conference on Tangible, embedded, and embodied interaction - TEI '11. ACM Press, New York, New York, USA, 129. https://doi.org/10.1145/1935701. 1935727

[46] Steve Nebel, Sascha Schneider, and Günter Daniel Rey. 2016. Mining Learning and Crafting Scientific Experiments: A Literature Review on the Use of Minecraft in Education and Research. Source: fournal of Educational Technology \& Society 19, 192 (2016), 355-366. http: //www.jstor.org/stable/jeductechsoci.19.2.355

[47] Richard E. Nisbett and Timothy D. Wilson. 1977. Telling more than we can know: Verbal reports on mental processes. Psychological Review 84, 3 (may 1977), 231-259. https://doi.org/10.1037/0033-295X.84.3.231

[48] Kimberly G. Noble, Bruce D. McCandliss, and Martha J. Farah. 2007. Socioeconomic gradients predict individual differences in neurocognitive abilities. Developmental Science 10, 4 (jul 2007), 464-480. https://doi.org/10.1111/j.1467-7687.2007.00600.x

[49] Adam C. Oei and Michael D. Patterson. 2015. Enhancing perceptual and attentional skills requires common demands between the action video games and transfer tasks. Frontiers in Psychology 6 (feb 2015), 113. https://doi.org/10.3389/fpsyg.2015.00113

[50] Lynn Okagaki and Peter A. Frensch. 1994. Effects of video game playing on measures of spatial performance: Gender effects in late adolescence. Fournal of Applied Developmental Psychology 15, 1 (jan 1994), 33-58. https://doi.org/10.1016/0193-3973(94)90005-1

[51] Eleanor O'Rourke, Kyla Haimovitz, Christy Ballweber, Carol Dweck, and Zoran Popović. 2014. Brain points: a growth mindset incentive structure boosts persistence in an educational game. In Proceedings of the 32nd annual ACM conference on Human factors in computing systems - CHI '14. ACM Press, New York, New York, USA, 3339-3348. https://doi.org/10.1145/2556288.2557157

[52] Nadia Oxford. 2018. What's the Definition of an Action Game? https: //www.lifewire.com/nintendo-action-game-1126179

[53] M Peters, B Laeng, K Latham, M Jackson, R Zaiyouna, and C Richardson. 1995. A Redrawn Vandenberg and Kuse Mental Rotations Test - Different Versions and Factors That Affect Performance. Brain and Cognition 28, 1 (jun 1995), 39-58. https://doi.org/10.1006/BRCG.1995.1032 
[54] Kasey L Powers, Patricia J Brooks, Naomi J Aldrich, Melissa a Palladino, and Louis Alfieri. 2013. Effects of video-game play on information processing: a meta-analytic investigation. Psychonomic bulletin \& review 20, 6 (2013). https://doi.org/10.3758/s13423-013-0418-z

[55] Eugene F. Provenzo, Jr. 1991. Video Kids: Making sense of Nintendo. Harvard University Press, Cambridge, MA and London, England. https://doi.org/10.4159/harvard.9780674422483

[56] Claudia Quaiser-Pohl, Christian Geiser, and Wolfgang Lehmann. 2006. The relationship between computer-game preference, gender, and mental-rotation ability. Personality and Individual Differences 40, 3 (2006), 609-619. https://doi.org/10.1016/j.paid.2005.07.015

[57] Claudia Quaiser-Pohl and Wolfgang Lehmann. 2002. Girls'spatial abilities: Charting the contributions of experiences and attitudes in different academic groups. British fournal of Educational Psychology 72, 2 (jun 2002), 245-260. https://doi.org/10.1348/000709902158874

[58] Katharina Reinecke and Krzysztof Z. Gajos. 2015. LabintheWild: Conducting Large-Scale Online Experiments With Uncompensated Samples. In Proceedings of the 18th ACM Conference on Computer Supported Cooperative Work \& Social Computing - CSCW'15. ACM Press, New York, New York, USA, 1364-1378. https://doi.org/10.1145/2675133. 2675246

[59] Bethany Rittle-Johnson, Robert S. Siegler, and Martha Wagner Alibali. 2001. Developing conceptual understanding and procedural skill in mathematics: An iterative process. Journal of Educational Psychology 93, 2 (2001), 346-362. https://doi.org/10.1037/0022-0663.93.2.346

[60] Giovanni Sala, K. Semir Tatlidil, and Fernand Gobet. 2017. Video Game Training Does Not Enhance Cognitive Ability: A Comprehensive Meta-Analytic Investigation. Psychological Bulletin (dec 2017). https: //doi.org/10.1037/bul0000139

[61] José Luis González Sánchez, Francisco Luis Gutiérrez Vela, Francisco Montero Simarro, and Natalia Padilla-Zea. 2012. Playability: analysing user experience in video games. Behaviour \& Information Technology 31, 10 (oct 2012), 1033-1054. https://doi.org/10.1080/ 0144929X.2012.710648

[62] Valerie J. Shute, Matthew Ventura, and Fengfeng Ke. 2015. The power of play: The effects of Portal 2 and Lumosity on cognitive and noncognitive skills. Computers and Education 80 (2015). https: //doi.org/10.1016/j.compedu.2014.08.013

[63] Valerie K. Sims and Richard E. Mayer. 2002. Domain specificity of spatial expertise: the case of video game players. Applied Cognitive Psychology 16, 1 (jan 2002), 97-115. https://doi.org/10.1002/acp.759

[64] Kurt D. Squire. 2008. Video Games and Education: Designing Learning Systems for an Interactive Age. Educational Technology 48, 2 (2008), 17-26. https://www.jstor.org/stable/44429558

[65] Kaveri Subrahmanyam and Patricia M. Greenfield. 1994. Effect of video game practice on spatial skills in girls and boys. Fournal of Applied Developmental Psychology 15, 1 (jan 1994), 13-32. https: //doi.org/10.1016/0193-3973(94)90004-3

[66] Penelope Sweetser and Daniel Johnson. 2004. Player-Centered Game Environments: Assessing Player Opinions, Experiences, and Issues. In International Conference on Entertainment Computing. Springer, 321332. https://doi.org/10.1007/978-3-540-28643-1_40

[67] Melissa S. Terlecki and Nora S. Newcombe. 2005. How Important Is the Digital Divide? The Relation of Computer and Videogame Usage to Gender Differences in Mental Rotation Ability. Sex Roles 53, 5-6 (sep 2005), 433-441. https://doi.org/10.1007/s11199-005-6765-0

[68] Melissa S. Terlecki, Nora S. Newcombe, and Michelle Little. 2008. Durable and generalized effects of spatial experience on mental rotation: gender differences in growth patterns. Applied Cognitive Psychology 22, 7 (nov 2008), 996-1013. https://doi.org/10.1002/acp.1420

[69] Nash Unsworth, Thomas S. Redick, Brittany D. McMillan, David Z. Hambrick, Michael J. Kane, and Randall W. Engle. 2015. Is Playing
Video Games Related to Cognitive Abilities? Psychological Science 26, 6 (2015). https://doi.org/10.1177/0956797615570367

[70] David Uttal and Cheryl Cohen. 2012. Spatial Thinking and STEM Education: When, Why, and How? In The Psychology of Learning and Motivation. Elsevier. https://doi.org/10.1016/B978-0-12-394293-7. 00004-2

[71] David H. Uttal, Nathaniel G. Meadow, Elizabeth Tipton, Linda L. Hand, Alison R. Alden, Christopher Warren, and Nora S. Newcombe. 2012. The Malleability of Spatial Skills: A Meta-Analysis of Training Studies. Psychological Bulletin 139, 2 (mar 2012), 352-402. https://doi.org/10. 1037/a0028446

[72] Vero Vanden Abeele, Bob De Schutter, Luc Geurts, Stef Desmet, Jeroen Wauters, Jelle Husson, Lieven Van den Audenaeren, Frederik Van Broeckhoven, Jan-Henk Annema, and David Geerts. 2012. P-III: A Player-Centered, Iterative, Interdisciplinary and Integrated Framework for Serious Game Design and Development. In Serious Games: The Challenge. Springer, 82-86. https://doi.org/10.1007/978-3-642-33814-4_14

[73] Brian N Verdine, Roberta M Golinkoff, Kathryn Hirsh-Pasek, Nora S Newcombe, Andrew T Filipowicz, and Alicia Chang. 2014. Deconstructing building blocks: preschoolers' spatial assembly performance relates to early mathematical skills. Child development 85,3 (2014), 1062-1076. https://doi.org/10.1111/cdev.12165

[74] Daniel Voyer, Susan Voyer, and M. Philip Bryden. 1995. Magnitude of sex differences in spatial abilities: a meta-analysis and consideration of critical variables. Psychological Bulletin 117, 2 (1995), 250-270.

[75] Jonathan Wai, David Lubinski, and Camilla P. Benbow. 2009. Spatial ability for STEM domains: Aligning over 50 years of cumulative psychological knowledge solidifies its importance. Fournal of Educational Psychology 101, 4 (2009), 817-835. https://doi.org/10.1037/a0016127

[76] William R. Watson, Christopher J. Mong, and Constance A. Harris. 2011. A case study of the in-class use of a video game for teaching high school history. Computers \& Education 56, 2 (feb 2011), 466-474. https://doi.org/10.1016/J.COMPEDU.2010.09.007

[77] H. Wauck. 2017. Game features and individual differences: What makes a spatial skill training video game effective?. In International Conference on Intelligent User Interfaces, Proceedings IUI. https://doi. org $/ 10.1145 / 3030024.3038286$

[78] H. Wauck, G. Lucas, A. Shapiro, A. Feng, J. Boberg, and J. Gratch. 2018. Analyzing the effect of avatar self-similarity on men and women in a search and rescue game. In Conference on Human Factors in Computing Systems - Proceedings, Vol. 2018-April. https://doi.org/10.1145/3173574. 3174059

[79] H. Wauck, Z. Xiao, P.-T. Chiu, and W.-T. Fu. 2017. Untangling the Relationship Between Spatial Skills, Game Features, and Gender in a Video Game. In International Conference on Intelligent User Interfaces, Proceedings IUI. https://doi.org/10.1145/3025171.3025225

[80] Timothy D. Wilson and Daniel T. Gilbert. 2005. Affective Forecasting. Current Directions in Psychological Science 14, 3 (jun 2005), 131-134. https://doi.org/10.1111/j.0963-7214.2005.00355.x

[81] Carmen R. Wilson Van Voorhis and Betsy L. Morgan. 2007. Understanding Power and Rules of Thumb for Determining Sample Sizes. Tutorials in Quantitative Methods for Psychology 3, 2 (sep 2007), 43-50. https://doi.org/10.20982/tqmp.03.2.p043

[82] Z. Xiao, H. Wauck, Z. Peng, H. Ren, L. Zhang, S. Zuo, Y. Yao, and W.-T. Fu. 2018. Cubicle: An adaptive educational gaming platform for training spatial visualization skills. In International Conference on Intelligent User Interfaces, Proceedings IUI, Vol. Part F1351. https: //doi.org/10.1145/3172944.3172954

[83] Nick Yee. 2017. Beyond 50/50: Breaking Down The Percentage of Female Gamers By Genre. http://quanticfoundry.com/2017/01/19/ female-gamers-by-genre/ 